\title{
2 Pilot Support System: A Machine Learning Approach
}

\author{
David Watkins ${ }^{1}$, Guillermo Gallardo ${ }^{2}$ and Savio Chau ${ }^{3}$ \\ 1 AVIAGE SYSTEMS Advanced Design \& Technology, GE AVIC Civil Avionics Systems; \\ David.Watkins@aviagesystems.com \\ 2 AVIAGE SYSTEMS Advanced Design \& Technology, GE AVIC Civil Avionics Systems; \\ Guillermo.Gallardo@aviagesystems.com \\ 3 AVIAGE SYSTEMS Advanced Design \& Technology, GE AVIC Civil Avionics Systems; \\ Savio.Chau@aviagesystems.com
}

\begin{abstract}
Pilots can be one of the factors in many air traffic accidents. When one or both pilots are impaired (e.g. fatigue, drunk), disabled, capable but wrong-headed, don't have sufficient training, distracted, miscommunicate with the air traffic controller, or follow wrong instructions from the air traffic controller, the risk of accident will increase dramatically. In some of these cases, the risk can be mitigated by using big data and machine learning. The system will collect and analyze large amount of data about the state of the aircraft, e.g., the flight path, the immediate environment around the aircraft, the weather and terrain information, and the pilots' input to control the aircraft. Additional sensors such as eye tracking devices and biological monitor can also be added to determine the condition of the pilots. If the pilots' input do not match proper reaction to the situation or the pilots are impaired, the learning machine will first provide an advisory to the pilots. If both pilots are impaired or incapable, a warning will be sent to the flight attendants and air traffic controllers so that they can take appropriate actions. The learning machine will be trained by both accident database and an automatic training system.
\end{abstract}

Keywords: Avionics Systems; Machine Learning; Big Data; Deep Learning; Pilot Behavior Analysis; Eye Tracking; Automatic Training System

\section{Introduction}

The NTSB has noted over recent years through toxicology tests of pilots who were involved in fatal aviation accidents that there is increasing evidence of prescription drug use and a variety of over-the-counter (OTC) drugs. This includes drugs that are potentially impairing. Based on this information, it is reasonable to conclude that pilots may be using OTC or prescription drugs without realizing these drugs can cause impairment. Pilot impairment is significant to flight safety and accident risks.

Additionally, a NASA research project [3] reported on the safety issues associated with pilot distractions and interruptions. This, when coupled with a recently updated NTSB Safety Alert on Pilot Decision Making and Risk Management [5], shows a definitive safety risk associated with distractions, interruptions, and increased pilot workload.

Within the above referenced safety alerts and research, there are enough referenced accidents and fatalities to warranting another look at this problem. In short, there is a need to identify the various unsafe flight deck scenarios where the pilots may be less effective in their duties and provide them assistance when needed.

\section{Pilot Support System}

The NTSB frankly notes $[4,5]$ many of these pilot behavioral risks can be prevented. The FAA has provided guidance to pilots regarding these and similar safety risks along with pilot actions to help manage these risks, however, this does not mitigate the risk associated with unintentionally or 
intentionally non-compliant pilots. The exposure to these risks may decline with compliance, however, it does not reduce the actual risk encountered when the pilot is non-compliant. In these instances, it is important to have a means of actively managing the risk thereby ensuring a safer flight environment.

\subsection{Risk Management}

To better address and manage these risks, a Pilot Support System based on machine learning coupled with big data analytics may be used [1,2]. The overall objective of the Pilot Support System is to identify:

1. Inefficient or weak patterns

2. Efficient or strong patterns

The system identifies these patterns during the various phases of flight (e.g., take-off, climb, cruise, descend, and landing). It will identify impairment, distraction, fatigue, or other pilot inhibiting events as well as pilot enabling events. If the system identifies significant events, it will trigger an advisory alert to the pilot. If the situation becomes more urgent, the advisory will be elevated to a caution and then a warning. These advisories, cautions, and warnings will help the pilot take proper actions. If the system determines both pilots are impaired or incapable, a warning will be sent to the air traffic controllers so they can take appropriate.

\subsection{Data}

The pilot support system will collect and analyze large amounts of data, such as:

- Own-ship data

- Flight path information

- ATC communication

- Weather/environmental data surrounding the aircraft

- Terrain information

- Pilot feedback through controls

- GPS

- Pilots Data (e.g., age, gender, experience, education, levels of training, eye vision degradation levels, cultural background, etc.)

- Pilot interactions with flight deck systems

Other sensors may be used, such as gaze trackers and bio sensors, to collect data and maintain a safe flight environment.

Additionally, the Pilot Support System utilizes big data analytics to provide improvements in a number of other areas. For example, the data may be used to improve training, flight deck design, and help reduce the pilot's cognitive workload

When appropriate, it is possible to also alert the pilots when behaviors are beneficial to flight deck safety. Identifying positive behaviors has been proven to strengthen those behaviors better than punishment. While there are significant constraints to doing this within the flight deck, appropriate means within those constraints can be used to help the pilots and train good behaviors as well as avoid bad behaviors. In fact, the system may be utilized outside the flight deck within a flight simulation training environment and would utilize data collected in an operational flight deck. Utilizing the system in both an operational and training environment can only help to strengthen the system's ability to identify new pilots versus experienced pilots and thereby tailor the flight experience appropriately for the given pilots. 


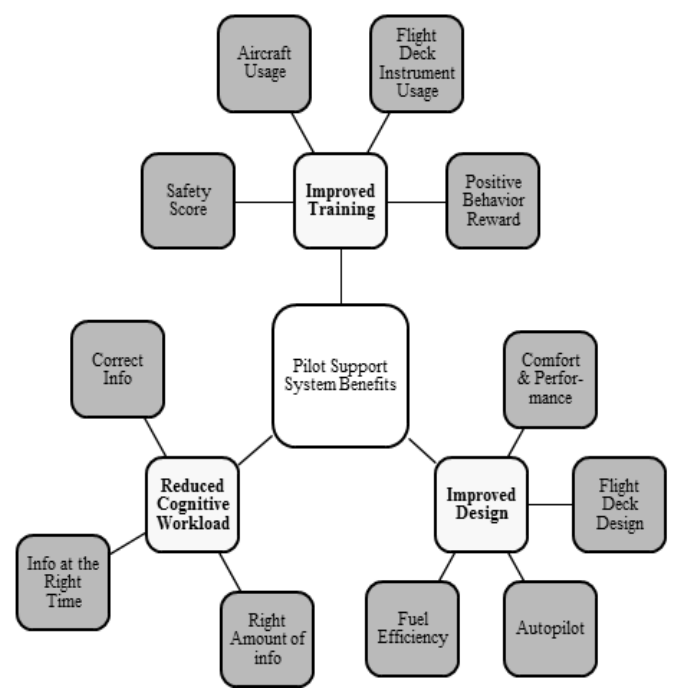

Figure 1. Pilot Support System Benefits

\section{Industry Benefits \& Value}

The benefits to this particular idea are almost innumerable, Figure 1. The ability to identify unsafe behaviors of those on the flight deck can lead to significant assurances of aircraft, flight crew, and passenger safety. The ability to train pilots can become more effective and enable airlines to train good habits in junior pilots. This will benefit not only the pilots but also the airlines due to instilling habits in the pilots which will help to reduce fuel costs on flights. With lower fuel costs, airlines will be able to provide lower cost flights for passengers which will increase traffic and income.

Additionally, aircraft manufacturers and avionics suppliers will benefit from the insights discovered by the pilot support system. This will enable the design of better flight decks, better human computer interfaces, and better overall avionics products. The information may lead to disruptive technologies which address problems on the flight deck in ways that truly meet the needs of the pilots, passengers, airlines, and industry. It can also help reduce engineering costs for those features that are unnecessary based on actual usage.

\section{Supporting Data and Analyses}

When discussing the functions of a Pilot Support System and how it may interact with pilots, it is important to also define in general terms the levels of a pilot's capability, Figure 2. These categories may be added to or modified as needed. This section discusses these categories [1].

\subsection{Capable but Distracted Pilots}

There have been a number of studies performed on the subject of distracted pilots. Whether pilots are performing necessary and expected tasks or they are involved with their personal electronic devices, a distracted pilot is an unsafe pilot.

Given the research performed on distracted pilots from both the 1998 NASA study and the 2003 Australian Traffic Safety Board study, a taxonomy of distracted pilots is continuing to evolve. The ATSB created their taxonomy due to limited information regarding actual distractions on the flight deck [8]. In other words, due to a lack of information, we are bounded in our classification and subsequent identification of distracted pilots.

However, the ATSB's classifications are useful for initial data collection as well as a continued review of the taxonomy of distracted pilots. This taxonomy will be the basis for ongoing efforts. 


\subsection{Insufficiently Trained Pilots}

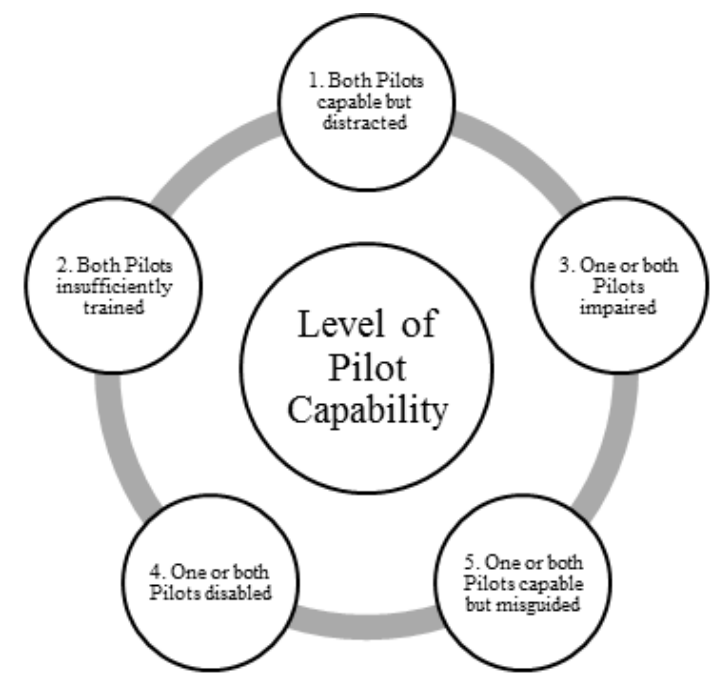

Figure 2. Use Case Scenarios

As a pilot develops greater proficiency in flights, there is a point at which a pilot must move from training to actual operation of a revenue flight. These types of transitions generally occur when pairing an experienced pilot with an inexperienced pilot. This may not always be possible in the future as the airline industry projects a shortage of pilots with respect to flight demand. Therefore, it will be essential to enable pilots placed into situations where they have limited training without the benefit of an experienced pilot.

The challenges found in this scenario surround the varying degrees associated with training. A pilot will have some training but may not be familiar specific flight scenarios. The number of flight scenarios could be very large. It will be important to identify the factors indicating when a pilot is less capable. There may be difficulties in having this category of pilot capability overlap with other capability categories. These are the challenges which need to be addressed as additional data are collected

Regardless of the varied flight scenarios, the best data collection point for identifying untrained or less trained pilots will be in real training scenarios. This can be in simulated flight scenarios as well as actual flight scenarios. In both instances, the system will collect and learn from pilot interaction during the major phases of flight and also how trainers provide correction to the pilot or aircraft to adjust for mistakes.

\subsection{Impaired Pilots}

This scenario may be more difficult than the others due to a potentially large overlap with untrained and distracted pilots. In fact, being distracted or untrained may be considered types of impairment. Similarly, there may be overlap where a pilot's cognitive capabilities are hindered due to impairment leading to a classification of the pilots as being misguided.

More definition will enable a better picture of this type of pilot behavior and a better means of classification and identification.

\subsection{Impaired Pilots}

Disabled pilots are those who are incapacitated and are unable to perform flight functions. This may be the easiest scenario to identify, for example, if the pilots are unresponsive to normal flight operations and alerts then the pilots may be safely categorized as disabled (e.g., normal required interactions on the flight deck are not performed over a significant period of time). 
The more difficult aspect of this will be determining the appropriate course of action. Is it sufficient to notify ATC? What if the communications equipment is not functional, will the system take control and maintain flight on behalf of the pilots as demonstrated recently by the Aurora Flight Systems Co-pilot? These are significant issues requiring further review.

\subsection{Misguided Pilots}

Perhaps the most difficult scenario to identify will be the misguided pilot. There are instances where the pilot is not distracted, not untrained, not impaired, and not disabled. In these cases, there may be instances where the pilot becomes confused and starts to make potentially unsafe decisions.

For example, if a pilot enters a cloud and becomes disoriented and begins to descend towards the ground during the cruise phase of flight, the system will be able to detect this kind of anomaly and provide feedback to the pilots to ensure the proper decisions are made.

Additional details will be obtained as data is collected, analyzed, and interpreted

\subsection{An Architecture of Pilot Monitoring System}

A Pilot Monitoring System is proposed to combat the aforementioned pilot frailties. This monitoring system is not intended to control or directly affect the flight operation. Rather, it will provide recommendations or reminders to the pilots at critical moments and alert flight attendance and air traffic control if necessary. The Pilot Monitoring System will have four functions: Pattern Recognition Function, Pilot Attention Monitor Function, Recommendation Function, and Alarm Function. The architecture is shown in Figure 3.

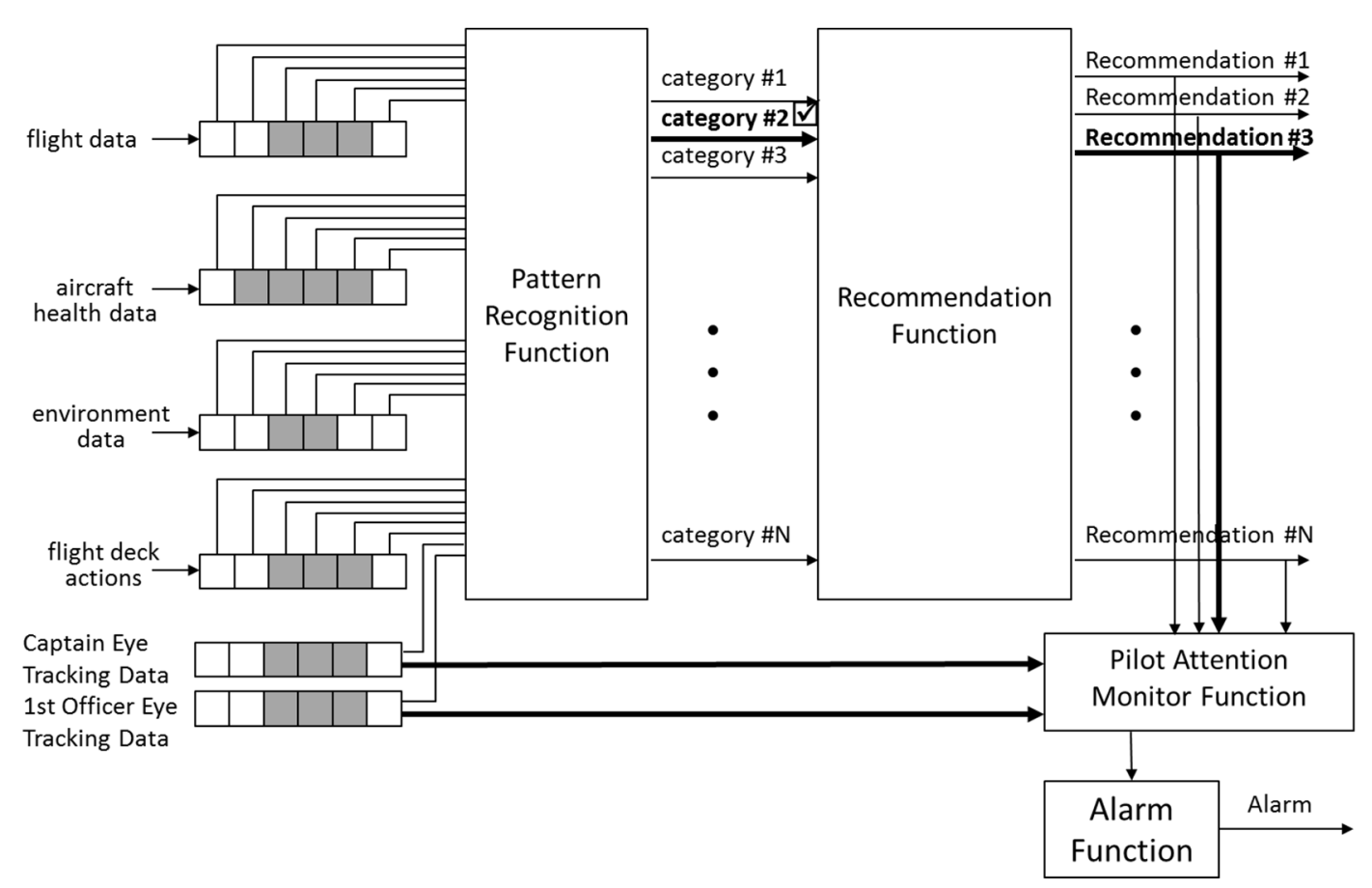

Figure 3. Notional Event Detection by Pilot Monitoring System

The Pattern Recognition Function is a learning machine which is trained to recognize event sequences that are similar to those prior to previous accidents. The event sequences include serial streams of flight data, aircraft health data, surrounding environment data, and pilots' flight deck actions. The learning machine is also trained to classify the previous accidents into categories. If the learning machine recognizes a pattern of streaming data that is similar to the event sequence prior to a previous accident, it will notify the Recommendation Function. The Recommendation Function will generate textual recommendations to the pilots based on the category. If the pilots do not agree with the recommendation, they have to explicitly reject the recommendation. 
In many cases, the pilots may not even pay attention to the text recommendations, as they may be fixated on one problem while ignoring the other warning signs. Therefore, it is important to determine whether the pilots' attention has been misplaced. The pilots' focus of attention can be detected by eye tracking technology [6]. When the Recommendation Function generates a recommendation, it will pass it to the pilot attention function. The Pilot Attention Monitor Function will first detect whether the pilots' eyes are reading the text recommendation. If the pilots are reading the text recommendation and they don't reject the recommendation, then the pilot Recommendation Function will track their eyes to see which instruments they are watching. If the pilots are not watching the text recommendation or they are not watching the instruments according to the recommendation, then an audible recommendation will be given. Again, the pilots have the ability to explicitly reject the recommendation if they prefer.

If the Pilot Attention Monitor Function cannot detect any eye movements, or the eyes of both pilots are closed, or both pilots are absent altogether, this may be an indication that both pilots are drowsy, incapacitated, or missing. This is a very serious problem. At this point, the Pilot Attention Monitor Function must notify the Alarm Function. The Alarm Function will take three actions. First, using the existing flight deck alert system, an alert will sound for pilot response. Second, the flight attendants will be alerted to check the pilots and address any medical situations as required (e.g., choking, heart attack, etc.). Third, the air traffic control will also be alerted and try to keep verbal communication with the pilots or give instructions to land the aircraft at the nearest airport.

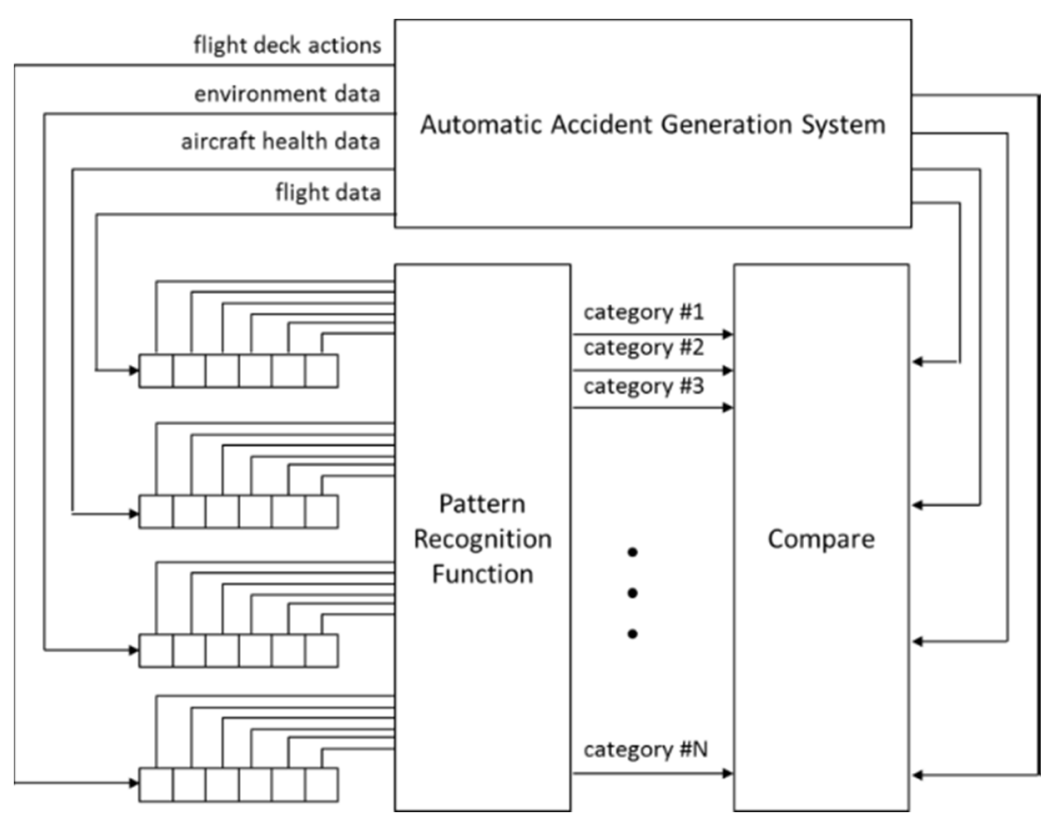

Figure 4. Training by Automatic Accident Generation

The most critical issue for pilot monitoring is to find sufficient data to train the Pattern Recognition Function. Accident data can be collected from the database of National Transportation Safety Board (NTSB) and other sources. Additional data can be generated by simulated accidents in pilot training. However, in order to get more training data, it is necessary to use an automatic accident generating and simulation system. This system will have a database of normal flight data and weather conditions along the flight path. Anomalies will be randomly injected to the normal flight data. The normal flight path will first be divided into five phases: taxing from the gate, climb, cruise, descent, and taxing to the gate. Each phase is divided into segments based on any changes in the flight path such as heading and speed. Then, a segment is randomly selected and a sequence of anomalies are injected into the data of the segment, including aircraft health data, environment around the aircraft, pilots' inputs and focus of attention, and other parameters. The flight path with injected anomalies is fed into a simulation model of the aircraft. If the injected anomalies cause an accident or near accident in the simulation, then the accident generation is successful. The 
successfully generated accident scenarios are used to train the pattern recognition function. As the automatic accident generating system knows what anomalies have been injected, it also knows the correct categorization of the accidents and the proper recommendation to be given to the pilots. When a simulated accident scenarios are fed to the input of the Pattern Recognition Function, but the Pattern Recognition Function cannot categorize the accident correctly, the error will be back propagated to the learning machine. This system is shown in Figure 4. The possible scenarios that can be generated by this automatic accident generation system can be infinite. The advantage is that it may discover potentially unknown accidents. Once the Pattern Recognition Function of one system has been trained, the knowledge can be replicated to other systems on all aircraft in the fleet.

\section{Conclusion}

There is a disconcerting trend in the industry towards more and more distracted or impaired pilots. Efforts to manage the risks need to include more than education of the pilots. Additionally, the efforts from NASA, NTSB, and ATSB have demonstrated there is insufficient data to accurately identify and classify the various distractors on the flight deck.

To address the data gap and provide a means to quickly act upon the insights obtained from the data, this proposed system which will collect data from the flight deck for the purpose of enabling pilots in their responsibilities and providing a natural means through which pilots may interact with the system. The system will fill the data void and enable a better, more accurate taxonomy upon which industry may rely to prevent further pilot distractions as well as address factors outside the pilot's control or capabilities.

\subsection{Future Work}

This paper is preliminary. As additional research and work is completed, more papers will be written providing additional details. The future work on this topic should include more accurate data collection and analytics to provide a clear picture of flight deck distractions and pilot behaviors. This effort must go beyond incident reports to better understand the drivers behind incidents and potential incidents. Additionally, it is necessary to determine which machine learning algorithms perform the best.

Also, continued effort to obtain data and understand the effects of human machine interaction on the flight deck are an integral part of ensuring mutually beneficial flight experiences and will be the basis upon which future avionics machine learning systems may be built.

\section{References}

1. H. Blair-Smith, "Ubiquitous big data vs. human frailty on the flight deck," 2014 IEEE/AIAA 33rd Digital Avionics Systems Conference (DASC), Colorado Springs, CO, 2014, pp. 2D5-1-2D5-9. DOI: 10.1109/DASC.2014.6979434.

2. John A. Caldwell, Melissa M. Mallis, J. Lynn Caldwell, Michel A. Paul, James C. Miller, and David F. Neri, "Fatigue Countermeasures in Aviation," Aviation, Space, and Environmental Medicine $x$ Vol. 80, No. 1 x January 2009.

3. Key Dismukes, Grant Young, and Robert Sumwalt, "Cockpit Interruptions and Distractions," Aviation Safety Reporting System (ASRS) Directline Issue Number 10: Dec 1998

4. "Pilots: understand Impairment Risk," NTSB Safety Alert, National Transportation Safety Board, SA-037 September 2014, revised December 2015.

5. "Pilots: Manage Risks to Ensure Safety," NTSB Safety Alert, National Transportation Safety Board, SA-023 March 2013, revised December 2015.

6. M. Reynal, Y. Colineaux, A. Vernay, and F. Dehais, "Pilot Flynig vs. Pilot Monitoring during the approach phase: an eye-tracking study," HCI-Aero '16, September 14 - 16, 2016, Paris, France. DOI: http://dx.doi.org/10.1145/2950112.2964583.

7. K. Kilingaru, J. Tweedale, and S. Thatcher, "Monitoring Pilot Situation Awareness," Journal of Intelligent $\mathcal{E}$ Fuzzy System vol 24, 2013. 
8 of 8

274 8. “Dangerous Distraction An examination of accidents and incidents involving pilot distraction in Australia 275 between 1997 and 2004," Australian Transport Safety Bureau, Aviation Research Investigation Report 276 B2004/0324, February 2006. 